Trauma Berufskrankh 2014 • 16[Suppl 2]:142-145 DOI 10.1007/s10039-013-2046-2

Online publiziert: 20. Februar 2014

(c) Springer-Verlag Berlin Heidelberg 2014

R. Fehse

Bezirksverwaltung Nord, BG BAU - Berufsgenossenschaft der Bauwirtschaft, Hannover

\title{
Schulterverletzungen aus Sicht der gesetzlichen Unfallversicherung
}

in $\$ 17$ Absatz 1 Sozialgesetzbuch I (SGB I) normierte Grundsatz der zügigen Leistungserbringung bezieht sich nicht nur auf die Durchführung des Verwaltungsverfahrens, sondern findet seine Erweiterung bei der Erbringung von Geld-, Dienst- und Sachleistungen [5].

Die Grundsätze des $\$ 17$ SGB I sind seit vielen Jahren erfolgreich in den Verfahren der Unfallversicherungsträger implementiert. Beispielhaft ist eine funktionierende Rettungskette zu nennen, beginnend mit der Ersthilfe am Unfallort, fortgeführt durch ein luft- und bodengebundenes Rettungswesen. Hier sind sämtliche Maßnahmen darauf ausgerichtet, Versicherte schnell einer zielführenden und umfassenden Heilbehandlung zuzuführen.

Bei der Ersthilfe gehen wir von einer hohen Struktur- und Prozessqualität aus. Allerdings sind statistische Daten hierzu kaum vorhanden. Aus diesem Grund wurden 45 Unfälle von Versicherten der Berufsgenossenschaft der Bauwirtschaft (BG BAU) mit Frakturen und Luxationen der Schultergelenke aus den Jahren 2010-2012 einer näheren Betrachtung zugeführt ${ }^{1}$.

In 64\% der der Untersuchung zugrunde liegenden Fälle wurde innerhalb der ersten Stunde nach dem Arbeitsunfall ein Durchgangsarzt (D-Arzt) aufgesucht, wobei die Zeit bis zur Vorstellung bei vielen Fällen deutlich unter $1 \mathrm{~h}$ lag. Insgesamt lässt dies darauf schließen, dass eine Ret-

\footnotetext{
1 Diese Fallmenge liegt allen Abbildungen in diesem Beitrag zugrunde.
}

tungskette mit hoher Qualität vorgehalten wird.

Darüber hinaus begegnen uns die Grundsätze des $₫ 17$ SGB I neben der zuvor beschriebenen Rettungskette an mehreren weiteren Stellen. So treten heute viele Unfallversicherungsträger auf ihren Internetseiten mit der Aussage an, dass sie schnelle und umfassende Hilfe für Betroffene anbieten. Krankenhäuser werben mit der Aussage einer schnellen, konsequenten und umfassenden Versorgung. Auch im Vertrag Ärzte-Unfallversicherungsträger sind Aussagen wie unverzüglich $(\$ 6)$, frühzeitig ( $\$ 17)$ und rechtzeitig $(\$ 18)$ enthalten. In diesen Kontext sind auch die Verfahrensabsprachen $\mathrm{zu}$ $\$ 14$ SGB IX einzuordnen [1].

\section{Durchgangsarztverfahren und Rehabilitation}

Das Durchgangsarztverfahren mit den hervorzuhebenden Merkmalen der Erstversorgung von Unfallverletzten und der Entscheidung über die Art der durchzuführenden Heilbehandlung (allgemeine oder besondere) hat zudem seine praktische Bedeutung darin, dass das aus dem Verfahren resultierende Berichtswesen in Form des Durchgangsarztberichts in aller Regel das Erstdokument darstellt, mit dem die Unfallversicherungsträger Kenntnis vom Eintritt eines Arbeitsunfalls erhalten ( $\bullet$ Abb. 1). Eine schnelle Berichterstattung durch den Durchgangsarzt versetzt damit den Unfallversicherungsträger in vielen Fällen überhaupt 


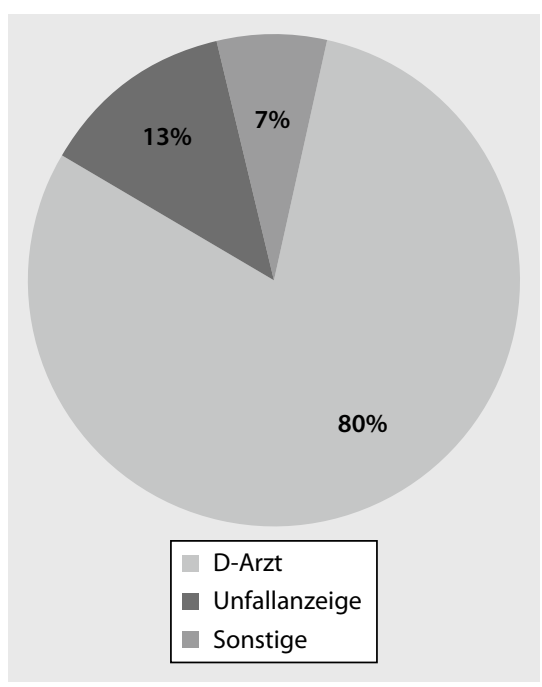

Abb. $1 \Delta$ Unfallmeldung (eigene Auswertung), D-Arzt Durchgangsarzt

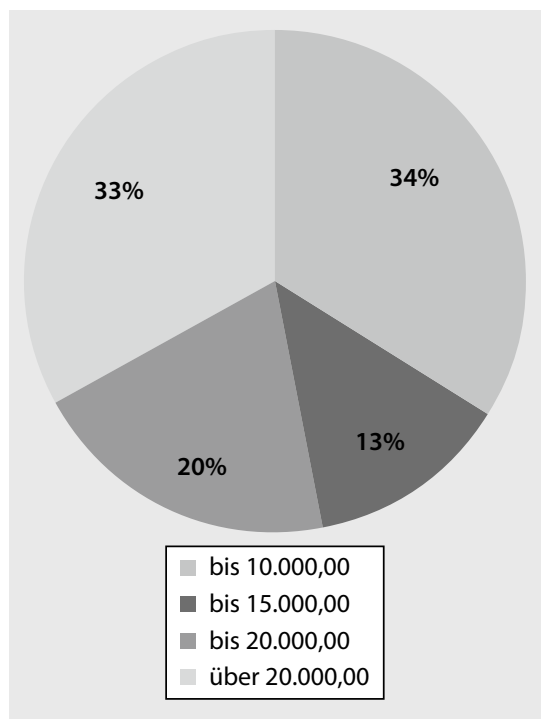

Abb. $3 \Delta$ Kosten pro Fall $[€]$ im ersten Jahr nach dem Unfall (eigene Auswertung)

erst in die Lage, zeitnah die den Versicherten zustehenden Leistungen zu erbringen bzw. auf Auskunftsersuchen sachgerecht antworten zu können.

Die Qualitätsanforderungen an die Rehabilitation von Schulterverletzungen aus Sicht der Unfallversicherungsträger beginnen mit der fachgerechten operativen Versorgung (soweit erforderlich) und beinhalten frühe rehabilitative Behandlungsansätze mit der korrekten Wahl der Belastungsintensität. Die Schnittstelle beim Übergang von der stationären in die ambulante Behandlung sollte so ausgestaltet sein, dass Versicherte idealerweise mit

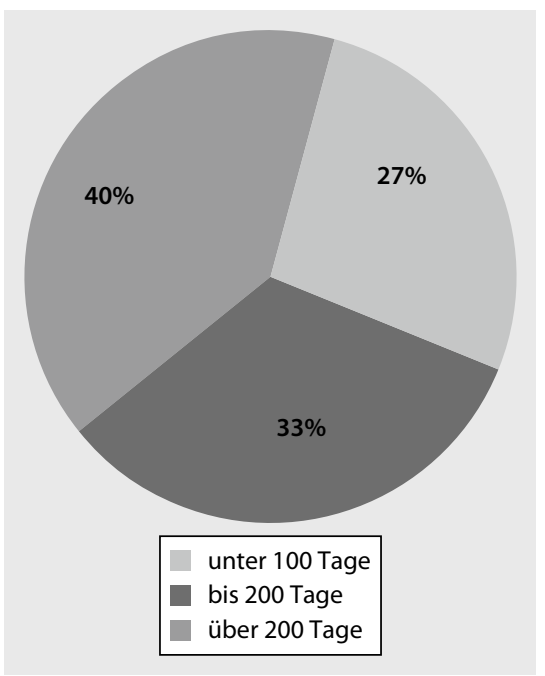

Abb. $2 \Delta$ Heilverfahrensdauer (eigene Auswertung)

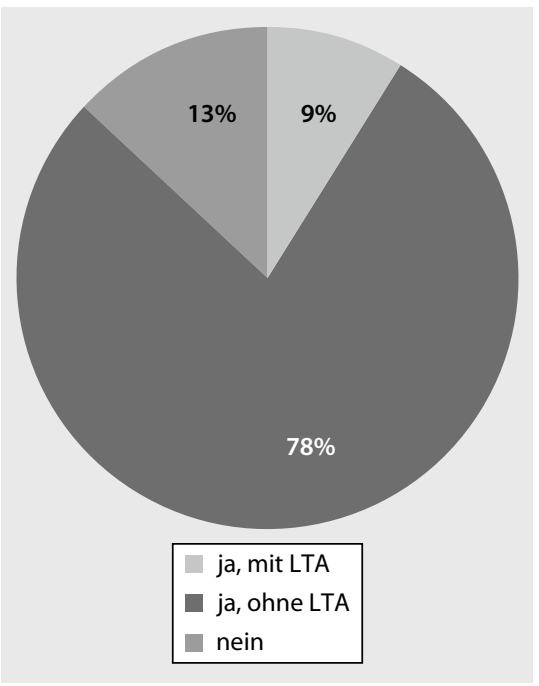

Abb. $4 \Delta$ Wiedereingliederung (eigene Auswertung), LTA Leistungen zur Teilhabe am Arbeitsleben

den Verordnungen und Berichten ausgestattet sind, die eine nahtlose Fortsetzung aller medizinischen und therapeutischen Maßnahmen ermöglichen.

Die Verläufe der Heilverfahren variieren breit, deutlich im Vordergrund steht mit 40\% der Fälle eine Dauer des Heilverfahrens von über 200 Tagen (D Abb. 2). Auch bei der finanziellen Betrachtung zeigt sich eine weite Verteilung. Hervorzuheben ist, dass in 33\% der Fälle Kosten von über 20.000,00 € im ersten Jahr nach dem Unfall entstehen (• Abb. 3).

\section{Rehabilitationsmanagement}

Nach dem Handlungsleitfaden der DGUV (Deutsche Gesetzliche Unfallversicherung) orientiert sich die Auswahl der Fälle, die dem Rehabilitationsmanagement zugeführt werden sollen, an einer prognostischen Arbeitsunfähigkeitsdauer für den Einzelfall [2]. Diese wird mit $112 \mathrm{Ta}$ gen beschrieben.

Die Grundsätze des Rehabilitationsmanagements beinhalten ein schnelles Aufgreifen der Fälle zur Einleitung und Steuerung der erforderlichen Maßnahmen, nach Möglichkeit schon mit der Erstmeldung eines Arbeitsunfalls durch den Arzt. - Abb. 2 mit der dargelegten Dauer des Heilverfahrens zeigt exemplarisch die Schwierigkeiten bei der Erstidentifikation im Rahmen des Rehabilitationsmanagements auf, die aus einer zeitlich vorgegebenen Prognose resultieren, denn die Prognosen und die tatsächlichen Verläufe bei Frakturen und Luxationen im Schulterbereich weichen deutlich voneinander ab und liegen $z$. T. unter- und oberhalb von 112 Tagen.

Zusätzlich zu dem beschriebenen Problem bei der Erstidentifikation von Fällen für das Rehabilitationsmanagement wird in der Praxis immer wieder evident, dass Diagnosen nach Erstattung des Durchgangsarztberichts einer Validierung unterliegen, was durchaus Auswirkungen auf prognostische Einschätzungen bezüglich des Verlaufs eines Falls haben kann. An dieser Stelle wird die Information durch den Arzt über (validierte) Diagnosen als Instrument für die Fallsteuerung für den Unfallversicherungsträger bedeutsam.

Die rechtzeitige und sachgerechte Einleitung rehabilitativer Maßnahmen beeinflusst Rehabilitationsverläufe bei Frakturen und Luxationen im Schulterbereich. Die funktionalen Ergebnisse, die durch die Rehabilitation erreicht werden, sind für die berufliche Wiedereingliederung entscheidend. Unsicherheiten bei prognostischen Abschätzungen zum Verlauf sollten deswegen dahingehend ausgelegt werden, im Zweifelsfall eher eine Zuordnung zum Rehabilitationsmanagement vorzunehmen - selbstverständlich mit der Möglichkeit einer späteren Korrektur. 
Sämtliche der Untersuchung zugrunde liegenden Fälle wurden durch ein Rehabilitationsmanagement begleitet. Die Wiedereingliederungsquote betrug 78\% (• Abb.4).

Das Rehabilitationsmanagement der Unfallversicherungsträger ist darauf ausgerichtet, die Wiedereingliederung von Unfallverletzten in ihre angestammte Tätigkeit zu erreichen. Dies gelingt trotz einer medizinischen Rehabilitation, die sich an dem Grundsatz „mit allen geeigneten Mitteln“ ausrichtet, nicht in allen Fällen. Der Anteil von Fällen, bei denen sich die Notwendigkeit von Leistungen zur Teilhabe am Arbeitsleben ergibt, liegt im hiesigen Verantwortungsbereich bei etwa 2\% der Fälle des Rehabilitationsmanagements. Bei den untersuchten Schulterverletzungen war er mit $9 \%$ deutlich höher. Dies wird darauf zurückgeführt, dass sich funktionale Beeinträchtigungen im Bereich der oberen Extremitäten gerade im handwerklich orientierten Gewerbe in besonderer Weise auswirken.

\section{Neuordnung der stationären Heilverfahren}

Schulterverletzungen werden sowohl im Verletzungsartenverfahren (VAV) als auch im Schwerstverletzungsartenverfahren (SAV) beschrieben [3]. Diese Regelungen sind seit dem 01.01.2013 wirksam, werden aber wegen der Realisierung der Rahmenbedingungen erst ab 2014 umgesetzt werden. In Bezug auf die Schulterverletzungen lassen die inhaltlichen Beschreibungen der Verfahren - vorbehaltlich der Praxiserfahrungen - auf eine verlässliche Umsetzung der vertraglichen Grundlagen zwischen Arzt und Unfallversicherungsträger schließen. Ein Nebeneffekt dieser Verlässlichkeit dürfte für Versicherte darin bestehen, dass sie in hohem Maß auf die Richtigkeit der Art der eingeleiteten Heilbehandlung vertrauen dürfen.

Die Neuordnung der stationären Heilverfahren erfordert eine Anpassung des derzeitigen Berichtswesens. Dies gilt sowohl für das SAV als auch für die Datenlieferung durch den Durchgangsarzt. Bisher stellen die Angaben zur AO-Klassifikation (AO: Arbeitsgemeinschaft für Osteosynthesefragen) im Durchgangsarztbericht kein Pflichtfeld dar. Die Aufnah- me der AO-Klassifikation an einigen Stellen im neuen Verzeichnis erfordert jedoch künftig konsequenterweise auch entsprechende Pflichtfelder im Durchgangsarztbericht.

Sehnenverletzungen in der Schulter waren in dem bis zum 31.12.2012 gültigen VAV-Katalog enthalten, sind aber im neuen Katalog für das VAV und SAV nicht mehr aufgeführt. Hier wird Ergänzungsbedarf gesehen!

\section{Begutachtung und Bescheid}

Auch bei Schulterverletzungen kann trotz optimaler Rehabilitation eine verbleibende Funktionseinbuße, sei es vorübergehender Art oder dauernd, nicht ausgeschlossen werden. Sofern die funktionalen Beeinträchtigungen auf eine vorübergehende Minderung der Erwerbsfähigkeit in rentenberechtigender Höhe hinweisen, wird heutzutage von einigen Unfallversicherungsträgern ein Rentenfeststellungsverfahren ohne Begutachtung praktiziert. Bei schweren Verletzungen, im Verlauf mit dauernder Funktionseinbuße, sollte zur Prüfung des Rentenanspruchs eine Begutachtung durch den Unfallversicherungsträger veranlasst werden. Beide Verfahren haben das gemeinsame Ziel, dass die Zeitspanne zwischen dem Eintritt der Arbeitsfähigkeit, der Begutachtung und der Bescheiderteilung möglichst gering sein soll.

Eine nähere Betrachtung der Prozessschritte im Falle einer Begutachtung macht deutlich, dass in aller Regel mehrere Zwischenschritte getätigt werden, in denen wiederum unterschiedliche Akteure eingebunden sind. Ein Regelablauf könnte sich wie folgt darstellen:

Der Meldung des Arztes zum Eintritt der Arbeitsfähigkeit folgt die Gutachterauswahl durch den Unfallversicherungsträger, die wiederum vom Versicherten zu beantworten ist. Dem dann folgenden Gutachtenauftrag durch den Unfallversicherungsträger folgt die Gutachtenerstattung durch den Arzt. Auf der Grundlage der Gutachtenauswertung wird die Beschlussfassung durch den Rentenausschuss vorbereitet, der wiederum zu festgelegten Terminen zusammenkommt. Danach kommt es zur Bescheiderteilung.
Trauma Berufskrankh $2014 \cdot$ - 16[Suppl 2]:142-145

DOI 10.1007/s10039-013-2046-2

(c) Springer-Verlag Berlin Heidelberg 2014

\section{R. Fehse}

\section{Schulterverletzungen aus Sicht der gesetzlichen Unfallversicherung}

\section{Zusammenfassung}

Versicherungsfälle allgemein. Eine schnelle Leistungserbringung ist in den Verfahren nach Versicherungsfällen von besonderer Bedeutung. Dies beginnt mit der Ersthilfe am Unfallort und setzt sich in der Steuerung durch den Durchgangsarzt, das Rehabilitationsmanagement der Unfallversicherungsträger bis hin zur Erteilung eines Bescheids fort.

Besonderheiten bei Schulterverletzungen. Ein Großteil der allgemein gültigen Grundsätze gilt auch für Schulterverletzungen, gleichwohl sind Besonderheiten und einige Neuerungen in den Abläufen zu beachten.

\section{Schlüsselwörter}

Zügige Leistungserbringung · Erste Hilfe . Durchgangsarztverfahren $\cdot$ Rehabilitation . Bescheiderteilung

\section{Shoulder injuries from the perspective of statutory accident insurance}

\section{Abstract}

Insurance cases in general. A rapid treatment process is particularly important in the procedure for insurance cases. This begins with first aid at the scene of the accident and continues in the guidance through the accident insurance consultant, rehabilitation management of the accident insurance company to the issuance of a decision.

Special characteristics of shoulder injuries. The majority of generally valid basic rules are also true for shoulder injuries; nevertheless attention must be paid to special features and some amendments in the procedures.

\section{Keywords}

Rapid treatment · First aid · Accident insurance consultant $\cdot$ Rehabilitation . Decision issuance

Für die für diesen Beitrag analysierten Fälle mit Schulterverletzungen konnte für die Zeit vom Eintritt der Arbeitsfähigkeit bis zur Bescheiderteilung unter Zugrundelegung aller Prozessschritte ein 
Mittelwert von 87 Tagen festgestellt werden. Eine nähere Betrachtung der einzelnen Prozessschritte legt durchaus Optimierungspotenziale offen. Dies gilt sowohl für den Unfallversicherungsträger bei den in seiner Verantwortung liegenden Maßnahmen als auch für den Arzt bzw. Gutachter.

Ob ein Mittelwert von etwa 3 Monaten bis zur Bescheiderteilung zufriedenstellend ist bzw. den Anforderungen des $\$ 17$ SGB I gerecht wird, soll an dieser Stelle offen bleiben.

Anzustreben ist in jedem Fall, dass Versicherte mit einem Rentenanspruch zeitnah zum Eintritt der Arbeitsfähigkeit den Rentenbescheid erhalten. Dies erfordert eine engmaschige Begleitung durch den Unfallversicherungsträger im Einzelfall. Die Vorgabe von realitätsnahen Zeitvorgaben als qualitätssichernde Maßnahme ist bei vielen Unfallversicherungsträgern durchaus Praxis. Zeitliche Vorgaben dürfen aber in keinem Fall dazu führen, dass die Qualität in der Begutachtung und die Richtigkeit der Bescheidinhalte nicht mehr den tatsächlichen Erfordernissen gerecht werden.

\section{Korrespondenzadresse}

\section{R. Fehse}

Bezirksverwaltung Nord, BG BAU -

Berufsgenossenschaft der Bauwirtschaft, Hildesheimer Straße 309, 30519 Hannover roland.fehse@bgbau.de

\section{Einhaltung ethischer Richtlinien}

Dieser Beitrag beinhaltet keine Studien an Menschen oder Tieren.

The supplement containing this article is not sponsored by industry.

\section{Literatur}

1. Bundesarbeitsgemeinschaft für Rehabilitation (2010) Gemeinsame Empfehlung über die Ausgestaltung des in § 14 SGB IX bestimmten Verfahrens. Bundesarbeitsgemeinschaft für Rehabilitation, Frankfurt am Main

2. Deutsche Gesetzliche Unfallversicherung (DGUV) (2010) Das Reha-Management der Gesetzlichen Unfallversicherung, Handlungsleitfaden. Rundschreiben Reha 0519/2010 vom 26.10.2010. DGUV, Berlin
3. Deutsche Gesetzliche Unfallversicherung (DGUV) (2012) Neuordnung der stationären Heilverfahren Rundschreiben Reha 0519/2012 vom 03.12.2012. DGUV, Berlin

4. Deutsche Gesetzliche Unfallversicherung (DGUV) (2012) Statistik Arbeitsunfallgeschehen 2011. DGUV, Berlin

5. Hauck K, Noftz W, Becker U, Knecht M (2005) Sozialgesetzbuch I: Allgemeiner Teil, Kommentar. Schmidt, Berlin, K § 17,3 